\title{
DESIGN SELECTION METHODOLOGY FOR COMPOSITE STRUCTURES
}

\author{
C. Monroy Aceves* A. A. Skordos M. P. F. Sutcliffe \\ Cambridge University Engineering Department, Trumpington St., Cambridge, \\ CB2 1PZ, U.K
}

\begin{abstract}
This paper presents a methodology to help designers select a shortlist or optimum design of composite structure from a large number of alternatives, taking into account conflicting design objectives or constraints (e.g. weight and cost). The methodology is based on creating a database containing results from an exhaustive search of a wide range of possible solutions. These results can be viewed using a commercial software selection package, originally written for materials selection. The designer then has freedom to change the selection criteria and required design constraints, to allow interactive selection of the data. The design methodology is illustrated by way of a case study, the design of a reinforced dogbone specimen.
\end{abstract}

Key words: composite design, structures, finite element analysis, optimisation, material selection, visualisation.

\section{Introduction}

Rapid advances in finite element (FE) methods have allowed their use as part of an optimisation strategy for design of composite structures. The route pursued by most researchers has been to use numerical optimisation strategies in conjunction with FE methods. While these numerical approaches are valuable where a single objective can be defined, they are less useful where there are conflicting design objectives (e.g. minimum weight and cost). Furthermore, most numerical optimisation techniques are not as good with discrete variables

* Corresponding author. Tel.: +44(0)1223 339883; fax: +44 (0)1223 332662

Email addresses: cm352@cam.ac.uk (C. Monroy Aceves), aas46@cam.ac.uk (A. A. Skordos), mpfs@eng.cam.ac.uk (M. P. F. Sutcliffe).

$\begin{array}{ll}\text { Preprint submitted to Elsevier Science } & 11 \text { July } 2006\end{array}$ 
(e.g. number of plies). Here graphical presentation of performance criteria al-

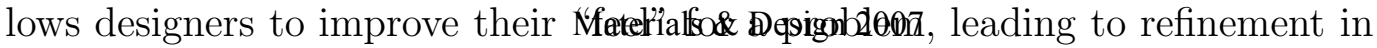
the design constraints and further design optimisation. Ashby $[2,4]$ describes how material performance indices (e.g. E/ $\rho$ ) can help with material selection, for a given design objective such as minimum weight or cost. The selection method can be facilitated using an 'Ashby map', for example plotting E as a function of $\rho$. The mapping idea can be extended via multiple selection stages, to take into account practical considerations [3]. The methodology developed by Ashby for materials selection provides a conceptual framework for selection of composite structures. The selection methodology can be used to choose between a wide range of variants of the proposed structure, with $\mathrm{FE}$ analysis providing the structural performance of the structure. The design selection methodology proposed here is suitable for the early stages of the design process, where the designer considers the widest possible range of solutions that will satisfy the constraints established in the design brief $[5,6,12]$.

Even when the problem is relatively simple, as with the dogbone case study described in this paper, the number of permutations that might be considered can be very large. Particular concerns with design optimisation of composite structures include: a restricted number of ply orientation angles should be considered; ply numbers must be integer; often (but not always) a balanced and symmetric lay-up should be used; several reinforcement regions can be included. When all these factors are taken into account, along with the need to consider different load cases and design objectives, tailoring the geometry, material properties and layup of the composite laminate to find an optimum design is very challenging $[6,17,10,11]$. The work presented here is an alternative approach to complex numerical optimisation methods, and is based on the use of $2-\mathrm{D}$ charts to evaluate laminate performance $[2,3,17,10,13]$. The power of the approach lies not only with the simplicity with which lots of data can be visualised, but also the ease with which the designer can interact with the data. This makes it relatively easy to pose 'what-if' questions about changes in the design constraints or objectives and so identify attractive ways of re-formulating the problem.

Section 2 outlines the process of designing with composite materials, the problems that the designer faces and the requirements of a selection methodology that can help in the early stages of the design process. In Section 3 we present details of the selection methodology proposed. We explain the methodology, giving a detail description of the steps to follow. A case study is described in Section 4 which illustrates the methodology. Finally, in Section 5 we give a concluding discussion about the methodology presented and we suggest future work to improve the selection methodology. 
Figure 1 gives an overview of the design process for composite materials. This starts with the market need (what do we need to do and why do we need to do it?), followed by a conceptual design (the ideas, possible solutions, materials, lay-ups, etc.), preliminary design (sketches, a short list of possible solutions) and detailed design (working drawings, more accurate data, refined cost estimates, finite element analysis, coupon tests). The process ends with the development of a prototype, and finally, the production of the design.

In the early stages of the design process, the designer has to take key decisions that will lead to the success or failure of the design. The designer has to select appropriate materials from within the materials universe. Where the designer decides to use composite materials, then further decisions are needed: which composite to use, which fibres with which resin, which manufacturing process, dry fabrics or pre-impregnated fabrics (pre-pregs), weaves or unidirectional fabrics, how many plies and in which orientations? These are some of the variables that the designer can play with in the early stages of the design process, and making a well-informed decision with so many options can be an overwhelming task. Hence any help that the designer can be given in quantifying these choices at this early stage is invaluable. A well informed decision at this stage will reduce the time needed in further design phases and help in optimising the final product.

The selection methodology presented in this paper is aimed to help the designer in this early stage, specifically, when the decision to use unidirectional pre-preg has been made.

One of the requirements set for the methodology was that it should be flexible to designer constraints. It was felt important to give the designer freedom to impose his/her constraints, and to change them on-the-fly. For this reason numerical optimisation techniques were avoided, as these do not easily allow a change in design constraints, without doing another time-consuming 'run'. From all the selection strategies available [4], the one used here is a "freesearch" strategy based on a quantitative analysis. This has the potential to be a fast, efficient and systematic way of giving innovative solutions. The freesearch quantitative analysis strategy works with a database, which should be structured in a hierarchical way. Normally a pre-stored database is used, but in our case thie database is built for each design problem, using FE analysis. 


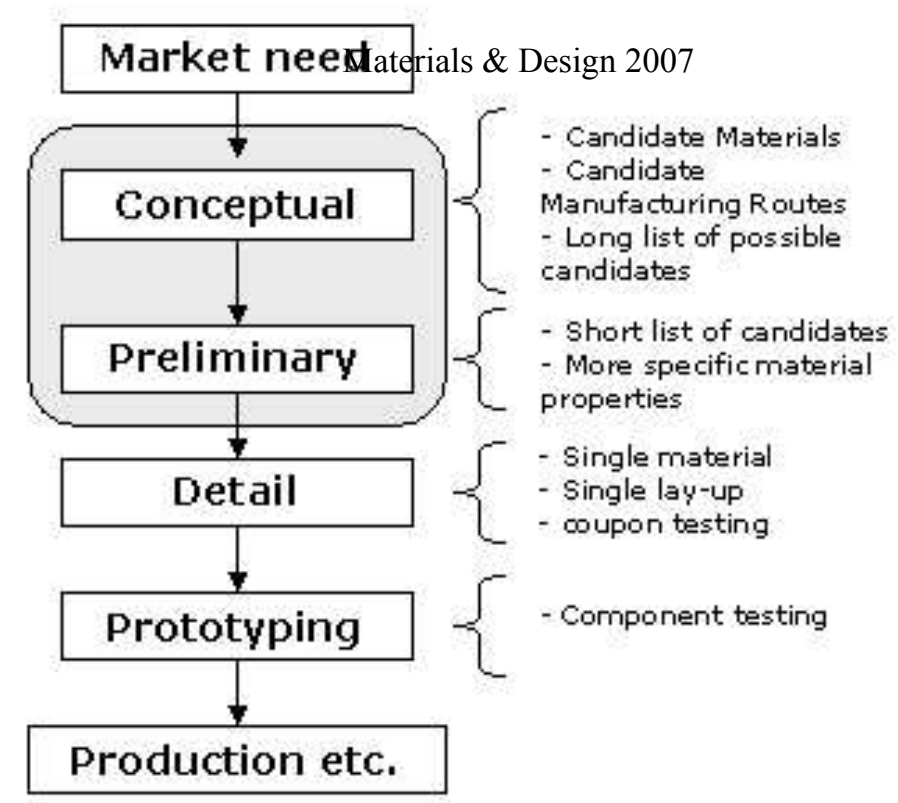

Fig. 1: Overview of the design process, after Ashby's work [5]

\section{Selection Methodology}

In this section we give an overview of the methodology and a step-by-step guide to the procedure. The following section gives a detailed description of how the methodology is implemented in a simple case study.

\subsection{Overview}

The basic idea of the methodology is to consider a very wide range of structural composite designs, perform appropriate calculations - including FE - to determine their performance, incorporate the results into a database, work with the data to screen out infeasible solutions or those not meeting the design constraints, and finally identify a shortlist of solutions which best meet the design goals. In essence the method builds on Weaver's methodology for laminate selection [17], but introduces structural analysis into the methodology, recognising the way in which structure and laminate lay-up are closely coupled in composites design.

Ashby's selection method has been implemented in user-friendly software, CES [13], which forms the selection 'engine' of the methodology. The CES software has the capability of manipulate the database in a user-friendly way, allowing the user to setup constraints to filter out the information in the database in order to make a selection. These constraints can be added via values or directly 
in a graph. What we do is create a database that has all possible solutions to the problem and manipulate/visuatisiselste idesign \$0017ables of design configurations and corresponding performance data are imported into a database within the software. The designer is then able to exploit the interactive capability of the selection software, to impose appropriate design constraints or selection criteria. To facilitate the process, and to help extract trends from the data, the records have been colour coded by laminate lay-up. Various performance windows shows the appropriate performance measures, such as stiffness or amount of material used. The designer can then use the software to straightforwardly impose constraints and optimise the component. For example, in the case study performance measures of deflection, cost and maximum strain energy are considered to identify a few appropriate lay-ups which fulfil the design requirements, while minimising use of material.

Ashby's selection methods and the CES methodology are well known and developed, and these have successfully been applied to material and process selection in mechanical design. The novelty of the work is to show how the selection-optimisation-visualisation tool can be used to meet the special challenges of designing with composite materials.

\subsection{Details of methodology}

Figure 2 gives a detailed flowchart of the design methodolgy. The procedure can be divided into six stages, as follows.

Design Brief. The design brief sets the framework for the design It gives the "need" that the product has to satisfy. The design brief has to answer (ideally all) the questions; What is the design for? Who is going to use it? How is going to be used? When? Where? How often? etc. The design brief has to give an idea of the batch size which will be produced. The design brief defines the requirements of the design. A simple example of a design brief could be: "Design a "high-tech" racing bicycle for the British team to use in the next Olympic games". From this design brief we can answer what do we have to do (a racing bicycle), who is going to use it (athletes), when and how often is going to be used (Olympic games and world class competitions), where it is going to be used (velodrome), batch size (between 10 and 20 bicycles), extra information (has to be very light!).

Definition of Problem In this stage it is important to identify clearly the constraints, variables and design objectives [3]. The information gathered in the design brief must be translated into quantitative constraints (e.g. maximum weight), variables and design objectives (e.g. minimum cost). We can use the quantify the information obtained for the design brief; using the bicycle example we can say that the load equals $80 \mathrm{~kg}$, corresponding 
to the average weight of an athlete. We could set a maximum weight to

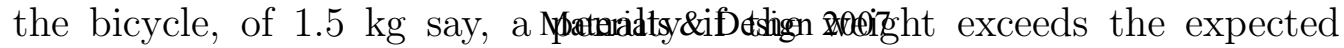
weight, or a premium if it is lighter. Here ranges over which the variables can change are set (for example specifying that only $0^{\circ}, 90^{\circ}$ and $\pm 45^{\circ}$ ply orientations can be used in the case of laminates). In this stage is where we define the size of the problem. By size of the problem we mean the number of possible combinations that we are going to analyze using finite element analysis. For example, consider that we can use only unidirectional prepreg fabrics and consider the case that we want to use only symmetric and balanced laminates of $0^{\circ}, 90^{\circ}$ and $\pm 45^{\circ}$ plies, varying the thickness of the laminate from 4 plies to 20 plies, we will be talking of a possible 140 cases per material. If we decide to try 3 different materials, we will be talking of 420 possible solutions. Therefore, the size of the problem can easily reach very big proportions even for a simple case. Hence the importance of defining in this stage the range and values that the variables can take. Laminate plate theory is then used to calculate material properties for all possible lay-up combinations.

Finite Element Analysis. At this stage the design is modelled using finite element analysis with MSC.Marc. Other analysis techniques could be appropriate here, but the emphasis is on real geometries and lay-ups, where the complexities lend themselves to FE analysis. In the early stages of the design process very accurate results are not needed to identify appropriate design concepts. Therefore a simple FE model, with modest run times, is sufficient. At a later stage in the design process the design methodology can be applied using a more sophisticated model, but with a smaller range of designs considered. A series of jobs is then submitted for analysis to cover all the parameter combinations to be considered (e.g. shape, material properties). Results are read between runs and the relevant output data saved to a database file. The template file is then modified and a new run submitted. The process is controlled by a code written in Microsoft Visual Basic, which takes the first input file, submits this input file for analysis into MSC.Marc, waits until the analysis has finished, then, it opens the output file, read the results and copy the appropriate values into the database that we are creating in Microsoft Excel. After this has been done, it modifies the input file, changing the variables or the properties we have set on the Definition of Problem, and submits this new input file for analysis. It does this until all possible combinations set in the previous stage had been analysed.

Create Database. A database is constructed containing records for all the design combinations considered, containing input data (geometry, laminate lay-up, etc) and corresponding results, including those from the FE analysis. The database is built using Microsoft Excel. The database has to be structured in a way that it can be imported into the selection / visualisation CES software.

Selection Process / Optimisation. The database is imported into the CES software. Constraints can now be applied to screen out inappropriate choices, 
the designs can be ranked using relevant performance indices and a shortlist or optimum design identified. Matheialss\&dessignaz0円7e repeated, modifying the limits and constraints, as needed.

Results. A shortlist of optimum solutions that meets the constraints is identified. Within this shortlist of solutions, the designer can choose design concepts that better suit their "feeling" of the problem, perhaps based on previous experience with composite materials, the infrastructure available to produce the design, available expertise in handling composites, etc. In this way judgement and analytical selection methods can be combined in a transparent way that can be easily explained to others in the design team. These chosen solutions can then go forward for further detailed design (e.g. using a more accurate FE model) and testing.

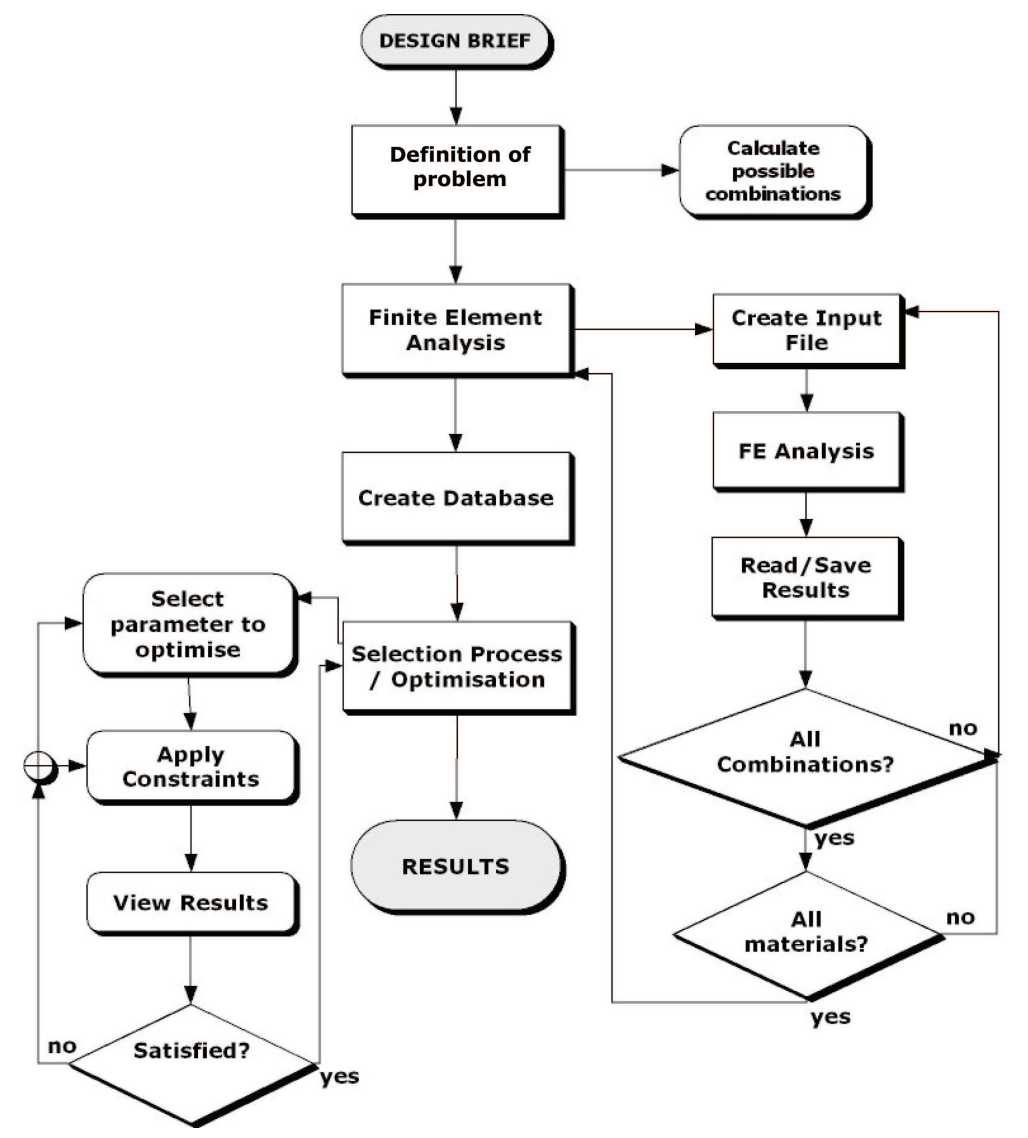

Fig. 2: Design methodology flowchart

\section{Application of the methodology - Dogbone Case Study}

The design methodology is illustrated by way of a case study. A dogbone specimen is considered, with a series of design constraints to simulate a practical composites design. This case study was chosen as it is simple but neverthe- 


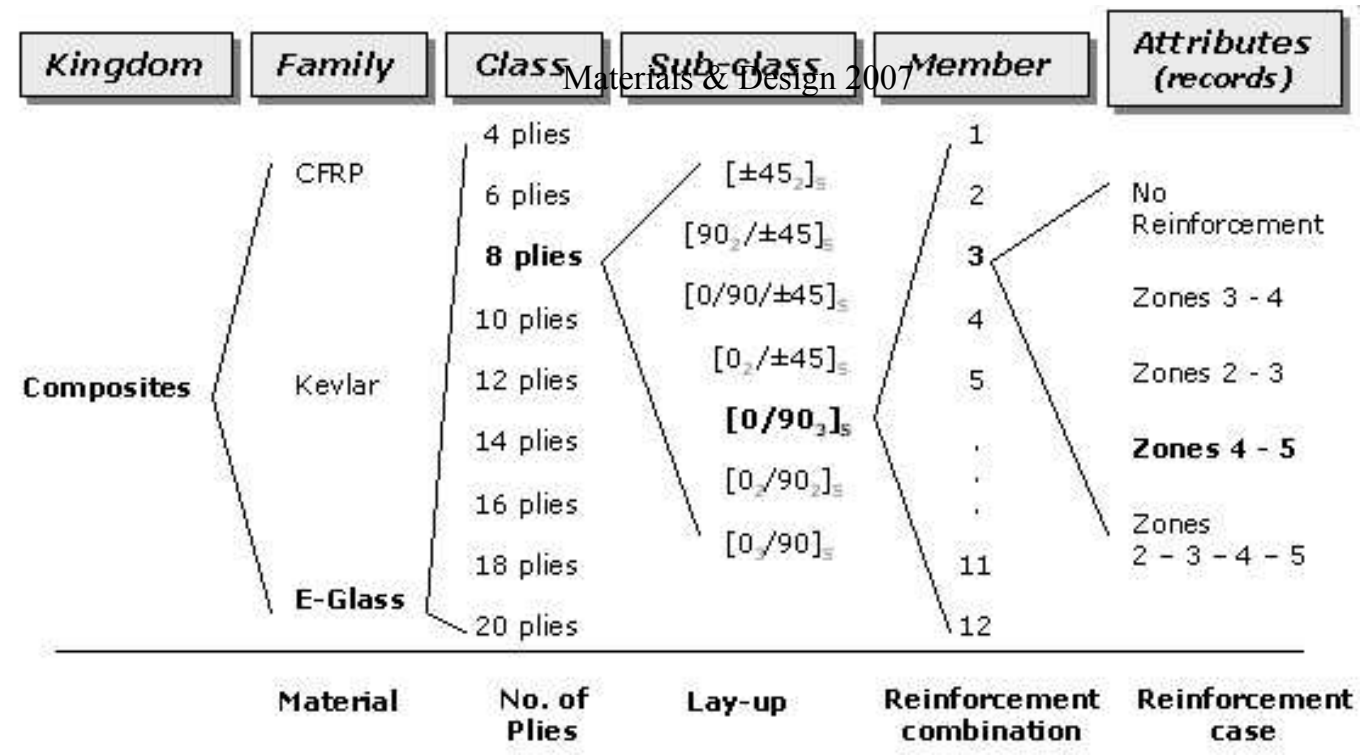

Fig. 3: The organization of the database in an hierarchical way

less allows consideration of the key challenges in design of composite design structures, including variable geometry, laminate lay-up and laminate reinforcement. The laminate lay-up is considered to be composed of a primary lay-up, which has the same ply orientations and thickness over the whole structure, and secondary reinforcement over part of the structure.

\subsection{Definition of problem}

\subsubsection{Geometry and loading}

The component, illustrated in Fig. 4(a) (which shows half of the symmetrical component), is of fixed length and width. Axial and shear loads are applied to the ends of the dogbone as indicated. In this study the radius $r$ of the cut-out is fixed at $11.67 \mathrm{~mm}$.

\subsubsection{Materials}

The specimen is made of unidirectional pre-preg from one of three materials; E-glass/epoxy, Kevlar/epoxy or carbon/epoxy. The properties of these materials can be found in Table 1 The thickness of the plies is $0.125 \mathrm{~mm}$. Only balanced symmetric lay-ups are considered (so that only half the thickness of the specimen needs be considered) and only ply orientations of $0^{\circ}, 90^{\circ}$ and $\pm 45^{\circ}$ are used. 


\subsubsection{Primary layup}

Materials \& Design 2007

For this case study, the layup has been considered as composed of a primary layup, which is constant over the whole specimen, and a secondary reinforcement lay-up over some areas. The total number of plies in the primary lay-up was varied from 4 to 20 , to give a total of 140 different combinations of $0^{\circ}$, $90^{\circ}$ and $\pm 45^{\circ}$ plies (considering that the laminate has to be balanced and symmetric).

\subsubsection{Secondary lay-up (reinforcement)}

The secondary reinforcement layup consists of extra plies, of the same material as the primary layup, applied to various combinations of zones on the specimen as illustrated in Fig. 4(a). The reinforcement can have as many as 3 plies per half of the laminate and it has to be balanced and symmetric (the same lay-up has to be added to the top and bottom of the laminate). This gives a total of 12 possible reinforcement combinations. Table 3 gives the 12 secondary lay-ups considered. Five different combinations of reinforcement zones are included, as detailed in Table 4 .

\subsubsection{Design objectives}

The design objectives which are considered in the problem are: weight, cost, deflection at the top-left node ' $A$ ' due to the applied load, and maximum strain energy density within the specimen. This latter objective is used as a simplified failure index. The deflection and the strain energy density are extracted from the FE output file for each run. The weight is calculated by finding the surface area of the part and multiplying it by the thickness and the density of the material. The cost is related only to the cost of the material. The cost is calculated by multiplying the mass of the component by the cost of the material per unit mass. The cost estimate used in this paper is for comparison purposes only and to allow cost to be included as a design variable. For a more accurate cost a more complex model needs to be used, such as those described in $[9,7,1]$.

The design constraints, variables and objectives used in the case study are summarised in Table 2. Considering the 140 combinations for the basic layup, the 12 possible combinations for the secondary lay-up and the five different reinforcement cases, we end up with 6860 independent cases for each material. With three different materials being considered, the number of independent cases increases to 20,580 .

The design of a dogbone specimen would seem at first sight to be very simple. However by including typical features of composites design, including choice 
Table 1: Material Properties (costs are very approximate)

\begin{tabular}{|l|l|l|l|l|l|l|}
\hline & $\begin{array}{c}\mathrm{E}_{11} \\
(\mathrm{GPa})\end{array}$ & $\begin{array}{c}\mathrm{E}_{22} \\
(\mathrm{GPa})\end{array}$ & $\begin{array}{c}\mathrm{G}_{12} \\
(\mathrm{GPa})\end{array}$ & $\begin{array}{l}\text { Poisson's } \\
\text { Ratio }\end{array}$ & $\begin{array}{l}\text { Density } \\
\left(\mathrm{kg} / \mathrm{m}^{3}\right)\end{array}$ & $\begin{array}{l}\text { Cost } \\
(£ / \mathrm{kg})\end{array}$ \\
\hline $\begin{array}{l}\text { E-glass/epoxy } \\
(\text { Scotchply/1002) } \\
\text { Kevlar/epoxy } \\
(\text { Kevlar 49/934) }\end{array}$ & 39 & 8.3 & 4.1 & 0.26 & 1900 & 5 \\
$\begin{array}{l}\text { Carbon/epoxy } \\
(\mathrm{T} 300 / 5208)\end{array}$ & 181 & 10.3 & 7.17 & 0.28 & 1500 & 100 \\
\hline
\end{tabular}

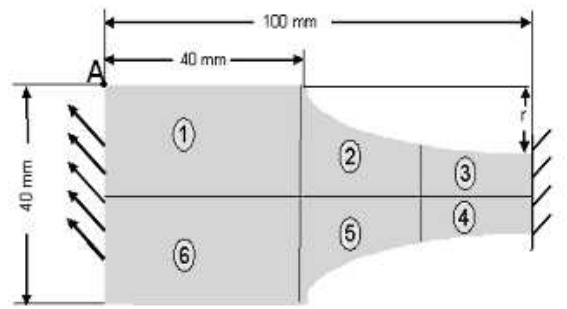

(a) Geometry

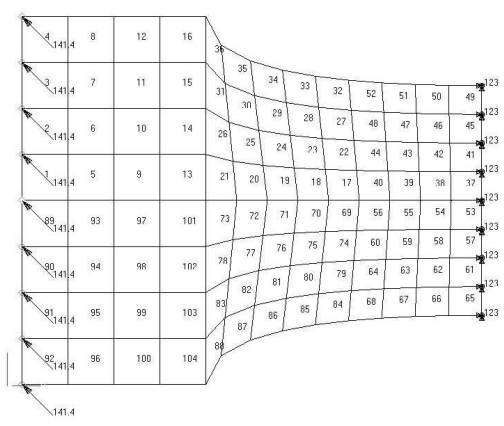

(b) FE model

Fig. 4: Dog-bone case study: (a) geometry; (b) finite element model

Table 2: Constraints, Variables and Design Objectives

\begin{tabular}{|l|l|}
\hline \multirow{2}{*}{ Constraints } & $\begin{array}{l}\text { Geometric: Fixed length and width } \\
\text { Loading: Fixed shear and axial load } \\
\text { Lay-up: Balanced, symmetric, } 0^{\circ}, 90^{\circ} \text { and } \pm 45^{\circ} \text { plies only }\end{array}$ \\
\hline Variables & $\begin{array}{l}\text { Material (CFRP, glass fibre, Kevlar) } \\
\text { Ply orientations and thicknesses in different regions }\end{array}$ \\
\hline Objectives & Weight, Cost, Deflection, Strain Energy Density \\
\hline
\end{tabular}

of material and lay-up, use of reinforcements and the constraint of using a discrete numbers of plies, it transpires that the designer is faced with a large number of possible solutions, exceeding 20,000 in this case. This wide choice, and the complications associated with anisotropic materials and geometric features, make it difficult to say a priori which design will be best. 


\subsection{Performance calculations and finite element analysis \\ Materials \& Design 2007}

A two dimensional finite element linear elastic model of the component was generated in MSC.Marc, as shown in Fig. 4(b). The tension and shear components of the pressure loading applied to the left-hand end of the component were $100 \mathrm{~N} / \mathrm{m}$, while symmetric boundary conditions were applied at the righthand end. Materials were treated as 2D orthotropic, with elastic properties $\left(E_{x}, E_{y}, G_{x y}\right.$ and $\left.\nu_{x y}\right)$ and material element thickness changed according to the primary and secondary lay-up used. The stiffness matrix of the material was obtained using laminate plate theory $[10,16,8]$. The model takes between 1 and 2 seconds to run. Each of the 20,580 possible designs was analysed using FE to give the maximum strain energy density and the displacement at node A.

\subsection{Performance plots}

The results were used to build a database, which was created with the tree structure shown in Fig. 5, adopting the tree structure approach used for the materials database of CES. Going from the general to the particular the structure consists of: a) Composites, b) Material (pre-preg, CFRP, E-glass, Kevlar), c) Number of plies in the basic lay-up, d) Possible ply combination to create a symmetric and balanced laminate, e) Reinforcement combinations (secondary lay-up) and finally, f) Reinforcement case (where a secondary lay-up is included).

With this database it is possible to create 2-D charts to compare the laminate performance using CES software. The results for the 20,580 independent cases are shown in Fig. 6. With the tree structure established in the data base, it is straightforward to confirm from Fig. 6(b) the obvious conclusion, that E-glass (pink) will be the cheapest solution, then Kevlar (blue), and finally carbon fibre will be the most expensive solution, but also the one with the best mechanical properties per unit mass. However Fig. 6(a) the Kevlar solution is reasonable where the displacement constraint is not too small. Some Kevlar solutions have displacements less than $0.5 \mathrm{~mm}$ and while there is not a signif-

icant increase in weight compared to carbon fibre specimens, there is a huge cost saving. The designer can readily see that, if the deflection constraint can be relaxed a little, choosing a Kevlar specimens will save a substantial amount of money (though a more sophisticated cost model would be needed to confirm this conclusion).

In summary, by using a tree structure and plotting the results with colour codes, for example by reinforcement combination or reinforcement case, the 


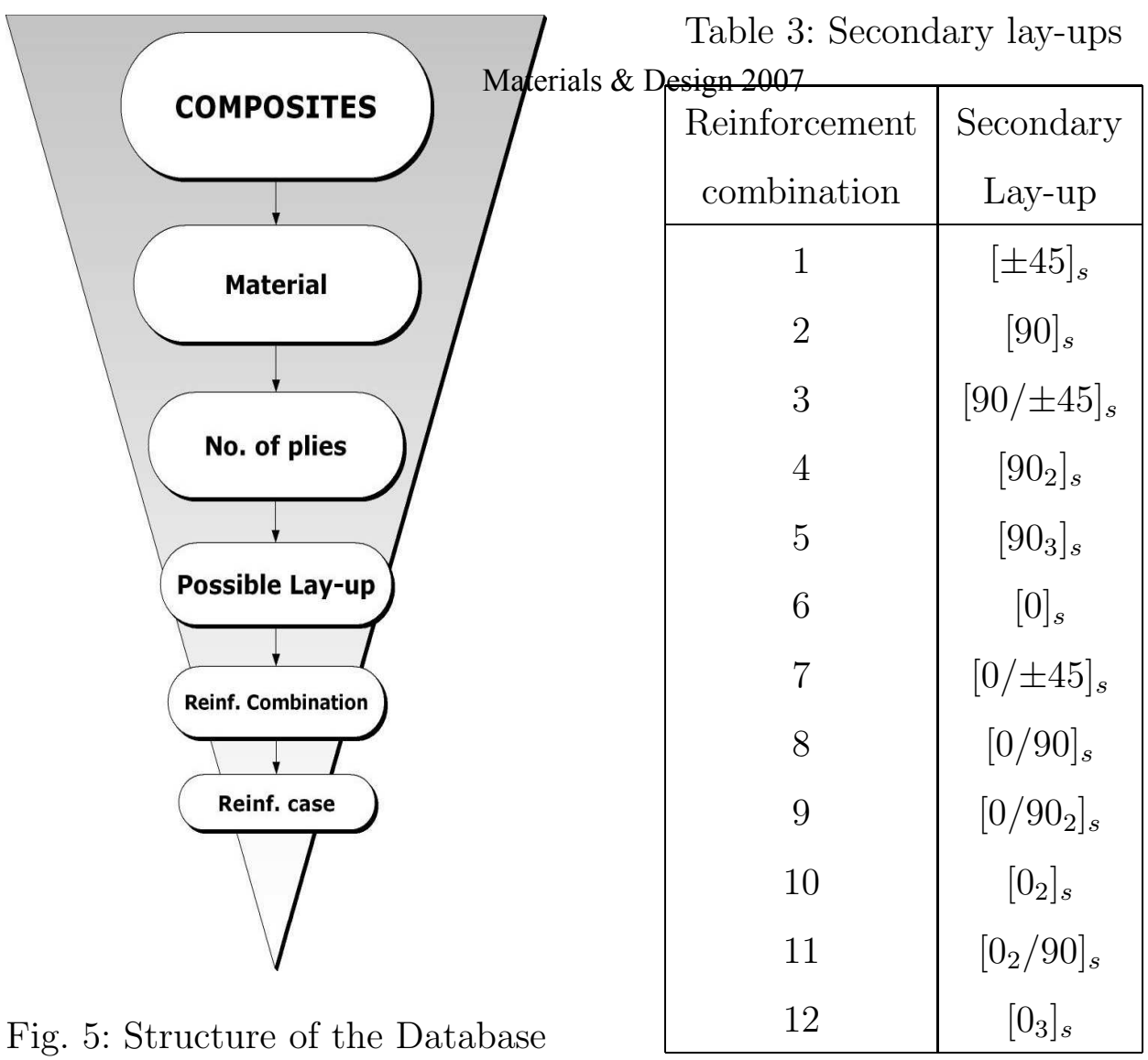

Table 4: Reinforcements zones

\begin{tabular}{|c|c|}
\hline Reinforcement case & Zones reinforced \\
\hline 0 & No reinforcement \\
1 & Zones $3-4$ \\
2 & Zones 2-3 \\
3 & Zones $4-5$ \\
4 & Zones 2-3-4 - 5 \\
\hline
\end{tabular}

selection software can extract useful trends or patterns that may exist in the results. In addition, appropriate constraints can be imposed to narrow the possibilities and search for the best solution, as illustrated in the next section.

\subsection{Selection}

In practical design, there will often be constraints which need to be applied to the design, perhaps arising from mechanical considerations or manufacturing. 
Here we show how these can be applied in an interactive way by the designer. The selection software allows thatdrisgzedebigmiditight areas on the performance plots within which the points must fall. For example, a box drawn in Fig. 7(a) imposes upper limits on the allowable deflection and mass. Because the various performance charts plotting the performance parameters are linked within the CES software, points lying outside the selected area on one selection chart are greyed-out on all the graphs. In other words, where different areas are selected on several graphs, only points passing all the selection criteria will remain active and coloured, as illustrated in Fig. 7(b).

To illustrate how this can be used, we include in the fictional design brief the following four design constraints:

- Cost $\leq £ 3$

- Mass $\leq 40 \mathrm{~g}$

- Displacement (at the top corner) $\leq 2 \mathrm{~mm}$

- Maximum strain energy density $\leq 2 \times 10^{6} \mathrm{~N} / \mathrm{m}^{2}$

When the data are represented graphically and the above constraints applied to the graphs (Figs. 7(a) and 7(b)) the number of cases is reduced to 3325. It would then be straightforward to identify suitable designs which meet all the constraints from these charts minimising mass or cost. However, to illustrate the flexibility of the selection method, consider applying additional constraints, perhaps arising from manufacturing considerations:

- The laminate must be quasi-isotropic, so that the primary layup is made of plies in the 4 directions $-0^{\circ}, 90^{\circ}$ and $\pm 45^{\circ}$.

- Reinforcement is applied only in zones 3 and 4 , if at all (reinforcement cases 0 or 1$)$

- A maximum of one extra ply of reinforcement is allowed per half laminate.

Applying these additional constraints gives only 38 possibilities as shown in Fig. 7(c) and 7(d). Of these 38, six correspond to E-glass, six to CFRP and the remaining 26 to Kevlar. All of these points have a laminate lay-up [0,90, \pm 45$]_{s}$ and have one or no reinforcement plies in zones 3 and 4 only. The optimal solution for each material has been found from the mass vs. deflection graph, as highlighted in Fig. 7(c). These same points have then been highlighted in Fig. 7(d). In Figs. 7(c) and 7(d), the cases that do not meet the constraints are not shown to aid visualisation. 

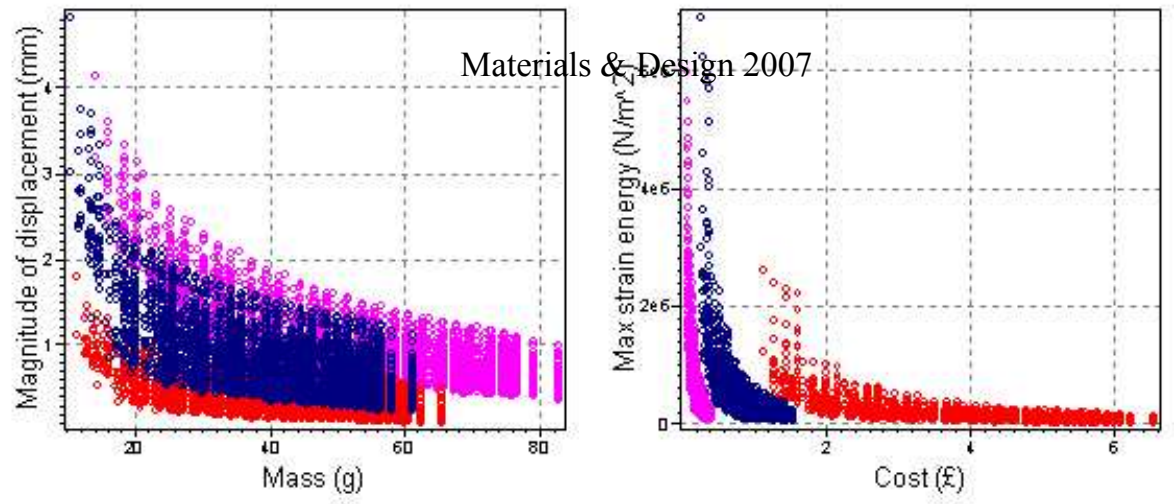

Fig. 6: Selection charts (magenta $=$ E-glass, blue $=$ Kevlar, red $=$ carbon fibre): (a) mass - deflection; (b) cost - strain energy
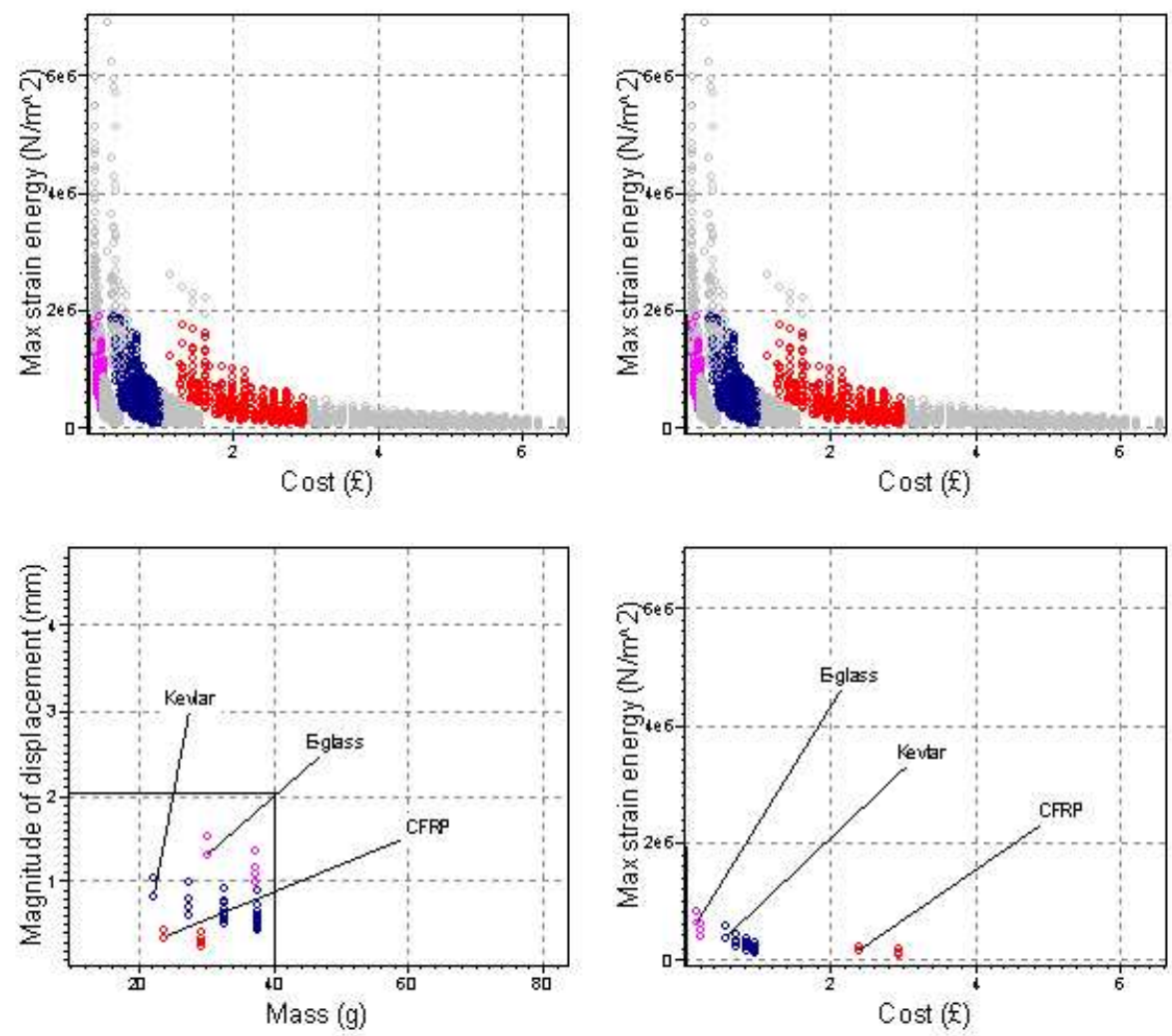

Fig. 7: Selection charts (magenta $=$ E-glass, blue $=$ Kevlar, red $=$ carbon fibre): (a) mass - deflection; (b) cost - strain energy (c) Optimum results; mass - deflection; (d) Optimum results; cost - strain energy

\section{Conclusions}

A simple design methodology has been developed, based on a methodology for material selection, to optimise composite structures. The method uses finite element analysis to identify the mechanical response of a range of proposed 
designs, and commercial selection software to impose appropriate constraints

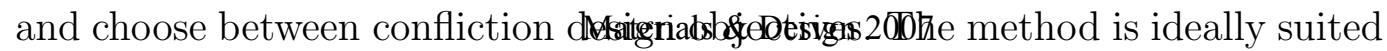
to more complex composite structures. Related work considers includung manufacturing constraints via a draping analysis. For these more complicated problems it is envisaged that an exhaustive search of the design space will not be feasible. Instead genetic algorithms can be used to generate a diverse population of proposed structures $[14,15]$.

The selection methodology for composite structures presented here is readily accessible by designers using composite materials. By facilitating substantial numerical modelling input at an early stage of the design process, it is possible for designers with less experience of working with composite structures to make sensible design choices at this stage. The method can also be used to help understand better the behaviour of composite structures and so develop a 'feel' for such problems. For example, the flexibility of the methodology can be used to learn from the database of results, identifying trends or patterns in the data that may not be visible otherwise. Finally, a family of databases for generic geometries with a range of load patterns could be built to save time in case of a redesign of a component.

\section{Acknowledgements}

The authors would like to acknowledge the support of: EPSRC, ESI Software SA, Ford Motor Company Ltd., Granta Design Ltd, Hexcel Composites, MSC.Software Ltd, University of Nottingham, Polynorm Plastics (UK) Ltd, Saint-Gobain Vetrotex International SAS, the U.K. Ministry of Defence and CONACyT - Mexico.

\section{References}

[1] P. Apostolopoulos and C. Kassapoglu. Recurring cost minimization of composite laminated structures - optimum part size as a function of learning curve effects and assembly. Journal of Composite Materials, Vol. 36(No. 4):501$518,2002$.

[2] M.F. Ashby. Materials Selection in Mechanical Design. ButterworthHeinemann, Oxford, 1999.

[3] M.F Ashby. Multi-objective optimisation in material design and selection. Acta Materialia, 48:359-369, 2000.

[4] M.F. Ashby, Y.J.M. Brechet, D. Cebon, and L. Salvo. Selection strategies for materials and processes. Materials and Design, 25:51-67, 2004. 
[5] M.F. Ashby and K. Johnson. Materials and Design; The art and Science of Material Selection in Product Materignds Butesigwodor-Heinemann, Oxford, 2002.

[6] M.G. Bader. Materials selection, preliminary design and sizing for the composite laminates. Composites: Part A, 27A:65-70, 1996.

[7] M.G. Bader. Selection of composite materials and manufacturing routes for cost-effective performance. Composites: Part A, 33:913-934, 2002.

[8] M.H. Datoo. Mechanics of Fibrous Composites. Elsevier Applied Science, UK, 1991.

[9] A.M.K. Esawi and M.F. Ashby. Cost estimation for process selection. Proceedings of ASME Design for Manufacture Conference (DETC99), Sept. 1999. Las Vegas, NV.

[10] Z. Grdal, R.T. Haftka, and P. Hajela. Design Optimisation of Laminated Composite Materials. John Wiley and Sons, Inc., USA, 1999.

[11] J.P. Leiva, D.K. Ghosh, and N. Rastogi. A new approach in stacking sequence optimization of composite laminates using genesis structural analysis and optimization software. AIAA, pages 1-8, September 2002. Symposium on Multidisciplinary Analysis and Optimization.

[12] A.V. Lotov, V.A. Bushenkov, and G.K. Kamenev. Interactive Decision Maps; Approximation and Visualization of Pareto Frontier. Kluwer Academic Publishers, USA, 2004.

[13] Granta Design Ltd. Ces selector, version 4.5. Cambridge, U.K., 2004. www.granta.co.uk.

[14] A.A. Skordos, C. Monroy Aceves, and M.P.F. Sutcliffe. Drape optimisation in woven composite manufacturing. In Proceedings of the 5th International Conference on Inverse Problems in Engineering: Theory and Practice, Cambridge, UK, 11-15th July 2005.

[15] A.A. Skordos, M.P.F. Sutcliffe, J.W. Klintworth, and J.P. Adolfsson. Multiobjective optimisation of woven composite draping using genetic algorithms. In 27th International SAMPE Europe Conference, Paris, France, 28 - 30th March 2006 .

[16] S.W. Tsai. Theory of Composites Design. Think Composites, USA, 1992.

[17] P.M. Weaver. On laminate selection and design. 43rd AIAA/ASME/ASCE/AHS/ASC Structures, Structural Dynamics and Materials Conference, pages 1-11, April 22 - 25 2002. Denver, CO. 